\title{
Topical Anesthetic-Induced Improvements in the Mobility of Patients with Muscular Hypertonicity: Preliminary Results
}

\author{
Mohamed A. Sabbahi and Carlo J. De Luca \\ NeuroMuscular Research Center, Boston University, Boston, Massachusetts, U.S.A.
}

\begin{abstract}
Summary: Application of a topical anesthetic on the skin of the upper and lower limbs of chronic stroke and head-trauma patients induced considerable improvement in limb mobility within $30 \mathrm{~min}$. We hypothesize that the augmentation of joint mobility and reduction in muscle rigidity are the result of desensitization of skin receptors that interact with the motor system. Physical therapy exercises performed during the effective period of the anesthetic rendered long-lasting improvement in the patients' ability to move their upper limbs and ambulate more effectively. Key Words: Motor control-Skin receptors-Skin desensitization-Hypertonicity-Rigidity-Topical anesthesia.
\end{abstract}

During the past several years, we have been exploring the use of topical anesthesia as a means of rehabilitating patients with muscular hypertonicity. We pursued this approach after previous studies of normal subjects demonstrated that monosynaptic reflexes (H-reflex and Achilles tendon reflexes ATR) could be modulated by desensitization of the skin $(10,12,13)$. Compatible results have been reported by other investigators working on related studies $(2,3,19,20)$. In our previous studies, desensitization of cutaneous receptors caused considerable facilitation of the H-reflex with no measurable change in the response of the ATR. A reduced sensitivity of the muscle spindle to stretch with a simultaneous facilitation in the excitability of the $\alpha$-motoneurons was cited as an explanation for these phenomena. Because the simultaneous effect

Accepted November 1, 1990.

Address correspondence and reprint requests to Dr. C. J. De Luca at NeuroMuscular Research Center, Boston University, 44 Cummington Street, Boston, MA 02215, U.S.A.

Dr. Mohamed A. Sabbahi is currently at the School of Physical Therapy, Texas Women's University, Houston, TX, U.S.A. on peripheral and spinal mechanisms suggested the use of a topical anesthetic for rehabilitation of movement in spastic patients, we investigated the immediate and long-term effects of skin desensitization in rehabilitation of patients affected with stroke.

\section{METHODS}

Nine patients (three women and six men) ranging in age from 21 to 84 years participated in the study. Eight were affected by stroke owing to cerebral vascular accidents (CVA), and one had head trauma. Computed tomography (CT) scans showed central nervous system (CNS) pathology at the cerebral cortex in four patients and at the internal capsule and basal ganglia in five patients. Four of the patients were afflicted with right motor hemiparesis, whereas five patients had left motor hemiparesis of varying degree. The patients were chosen according to the following criteria: their CNS lesion was at least 6 months old; they no longer showed noticeable improvement in physical performance, as reported by their physician and physical therapist; 
they were able to ambulate with or without an assistive device; and they retained normal superficial skin sensation in their affected limbs. Patients were not on medication when selected or throughout the study. Only seven of the nine patients were able to participate in the gait assessment segment of the experiment.

A battery of measurements was made on each patient: kinesiologic measurements of gait, videotape recordings of active movement patterns, time measurements of rapid repetitive movements (RRM) of the affected elbow and knee joints, and assessment of active and passive ranges of motion (ROM) of upper and lower limb joints. The measurements were obtained both before and after (at intervals $\leqslant 30 \mathrm{~min}$ ) application of a topical anesthetic ( $20 \%$ benzocaine) to all skin areas of the affected upper and lower limbs except the anterior tibial skin area $(10,12,13)$.

The immediate effect of the topical anesthetic on the recorded parameters was ascertained by comparing the results of the anesthetic spray tests to a second set of tests in which a placebo spray was applied on the same patients. The placebo spray was identical in all respects to the anesthetic spray except that it lacked the active ingredient. The longterm effect was determined by a 2-month singleblind study that combined a physical therapy program with application of the topical anesthetic or a placebo spray three times a week. For the first month, patients were selected at random to receive either the placebo or the anesthetic. During the second month, the procedure was alternated so that patients who received the anesthetic received the placebo and vice-versa. Thus, at the end of 2 months, each patient had received 1 month of anesthetic application and 1 month of placebo application.

Five minutes after its application, the topical anesthetic induced a partial reduction in skin sensation to superficial touch, which was ascertained by moving cotton wool along the skin. Deep sensation of pin prick, however, was not distinguishably different from normal sensation in the contralateral limb. This minor sensory diminution was not noted when the patient was sprayed with the placebo. $\mathrm{Pa}-$ tients also reported that their limb muscles were "looser," and that they had increased ability to control the direction and speed of limb movements after the topical anesthetic was applied.

Gait parameters were measured as the patient walked without shoes on a walkway in which a computer-controlled force platform was embedded. Three foot switches made of tin-foil paper were attached to the bottom of the patient's feet. Contacts were mounted under the heel and the heads of the first and fifth metatarsals. The walkway was covered with a metallic surface which served as the other contact for the switches. Time histories of the gait cycle and the vertical ground reaction forces during stance phase were recorded for subsequent analysis. Data from a total of 15-25 step cycles were recorded for each patient. Four parameters were used to describe the time histories of gait cycles: swing time, double limb support time, single limb support time, and cadence. These parameters were used to calculate the symmetry factor, which measures the time-ratio between two successive steps, and the temporal asymmetry index, which measures the asymmetry between the contralateral swing and double support times. The symmetry factor (SF) has been proposed by Knutsson (7) as a measure of the time-ratio between two successive steps. The equation is:

$\mathrm{SF}=\frac{\text { Left step time }}{\text { Right step time }}=1$ for normal gait.

The temporal asymmetry index (TAI) was proposed by Dewar and Judge (1) as a measure of the time duration of the swing and double support phases of the affected versus the nonaffected limbs. The equation is:

$$
T A I=\sqrt{\left(X_{R}-X_{L}\right)+\left(\mathrm{Y}_{\mathrm{R}}-\mathrm{Y}_{\mathrm{L}}\right)},
$$

where $X_{R}, X_{L}=$ the right and left double support times as percentage of stride time and $Y_{R}, Y_{L}=$ the right and left swing time as percentage of stride time. For normal subjects, this value ranges from 0 to 5; for hemiplegic patients, it ranges from 10 to 30 .

Gait parameters were analyzed with chi-square test to establish the significance of modifications associated with the anesthetic and placebo spray. Time measurements of $10 \mathrm{RRM}$ at the full joint range and performed between two fixed points were taken for the knee joint and the elbow joint. Three RRM tests were performed with rest intervals of 2 min. Movement sequences containing cycles that did not traverse the complete excursion range were discarded. The average value of the RRM tests was calculated. 


\section{RESULTS}

The results are discussed in two sections. The first section, Immediate Effects, describes observations made $45 \mathrm{~min}$ after first application of the anesthetic or placebo. The second section, LongTerm Effects, describes observations made at the end of the 1-month treatment program. In this second group, measurements were made without application of either anesthetic or placebo spray.

\section{Immediate Effects}

The improvement in movement parameters resulting from application of the anesthetic is evident in Figs. 1 and 2 and Tables 1 and 2 by comparison of the results with those of the placebo. We consider a change in the value of the parameters in the direction toward normalcy as an improvement.

Figures 1 and 2 show the improvement in the time required for 10 RRM at the elbow and knee joints for two representative patients. Figure 1 shows the results for the elbow joint of a representative patient. A reduction in performance time of $4 \mathrm{~s}$ or $20 \%$ occurred $45 \mathrm{~min}$ after anesthesia when the patient was initially tested. The time required to accomplish this test was decreased in varying amounts after each application of the anesthetic during the 1 -month period of the anesthesia program. When a $t$ test was performed between the pretest and posttest measurements, the difference was statistically significant $(\mathrm{p}<0.05)$ at days $1,9,11,13,16$, and 30 during the 1 month of anesthesia. Figure 2 shows similar results for the knee joint of another representative patient. A reduction in performance time of $1-4 \mathrm{~s}$ is evident $45 \mathrm{~min}$ after anesthesia throughout the second month, when the patient was treated with anesthesia. A statistically significant $(\mathrm{p}<0.05)$ reduction in performance time was recorded at days $4,9,14,22$, and 27 . These changes in performance time were not noted during the first month of treatment with placebo. Similar results were obtained for the knee and elbow joints in all patients. No measurable changes were noted immediately after each application of the placebo. This increase in movement velocity indicates an apparent reduction of muscular rigidity. This notion is consistent with observations made in our previous work $(15,16)$.

Table 1 shows the gait data for the seven patients who participated in this segment of the experiment. Table 2 shows the RRM data of all nine patients. A plus sign indicates an improvement of the average values toward the normal value. A minus sign indicates a deterioration of the average value. A blank (empty parentheses) indicates that the difference in the average values before and after application of the spray was $<15 \%$. This variability was deemed to represent the experimental error. An asterisk indicates that the difference was statistically significant at the $\mathrm{p}<0.05$ level. Generally, all parameters except TAI showed improvement $30-45 \mathrm{~min}$ after application of the anesthetic. The modifications noted after application of the placebo, however, were varied and considerably less pronounced.

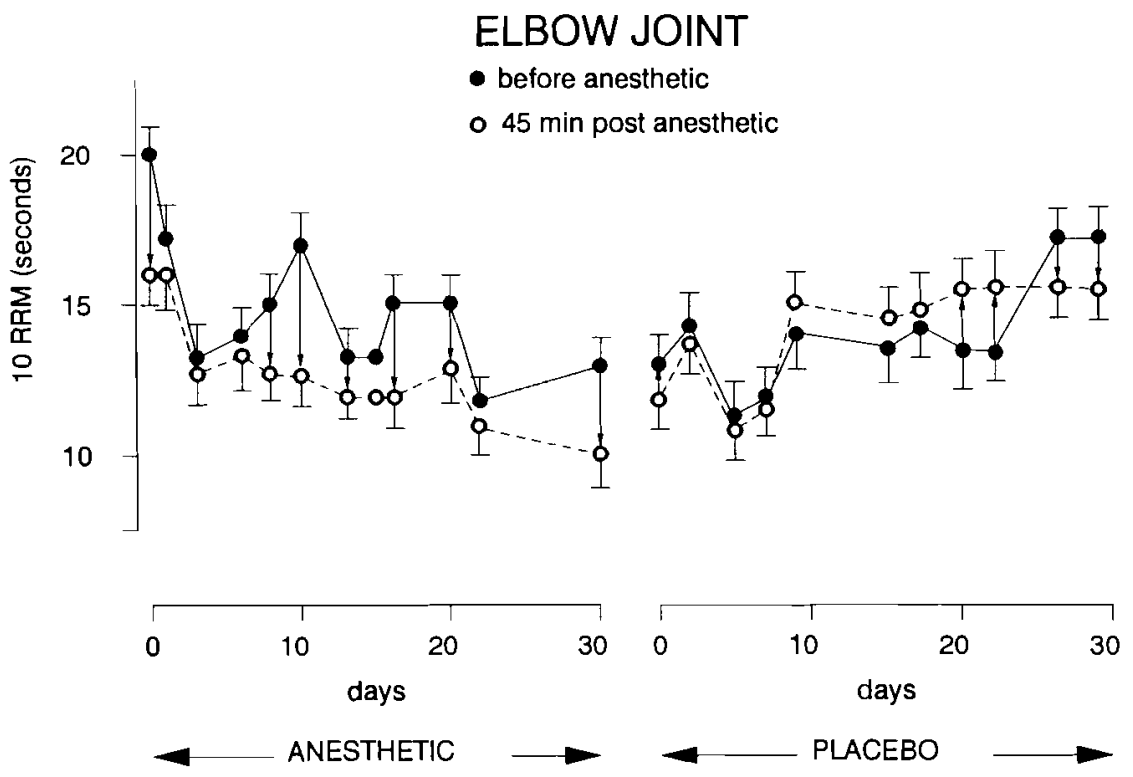

FIG. 1. Time measurement for completion of 10 rapid repetitive movements (RRM) at the elbow joint of the affected upper limb in a 60-year-old hemiplegic patient. Mean and standard deviation values of three sequences of measurement before application of topical anesthetic or placebo (solid symbols and lines); time measurements $45 \mathrm{~min}$ after application (open symbols and dashed lines). The amount of time required to accomplish the 10 RRM decreased immediately after each application of anesthetic but not application of placebo. Required time gradually diminished as a function of number of applications (day) of anesthetic but not of placebo. 
FIG. 2. Time measurement for completion of 10 rapid repetitive movements (RRM) at the knee joint of the affected lower limb in a 35-year-old hemiplegic patient. Mean and standard deviation values of three sequences of measurement before application of topical anesthetic or placebo (solid symbols and line); time measurements 45 min after application (open symbols and dashed lines).

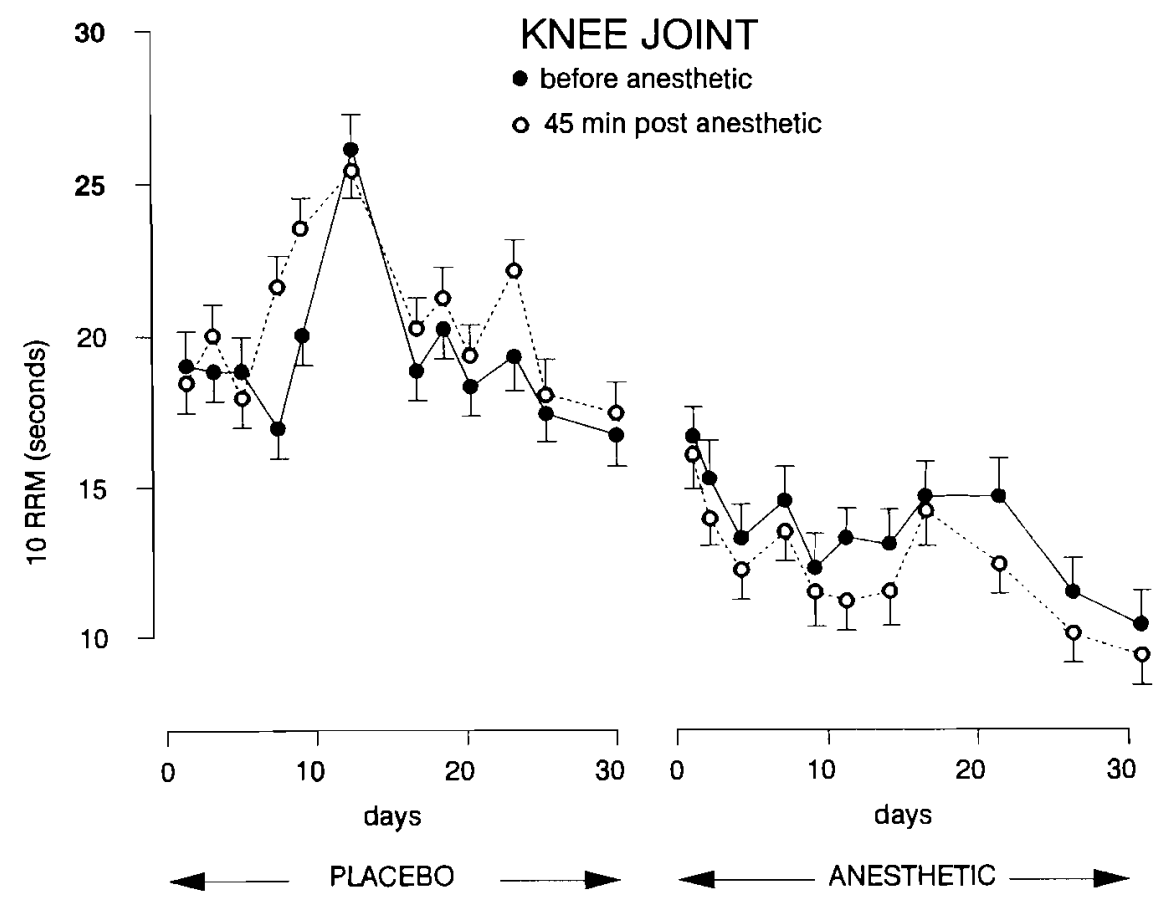

Modifications were noted in the vertical ground reaction forces. An example of the array of force vectors and the force envelope of a normal excursion of the foot on the force plate is presented in Fig. 3a. Comparable data from a stroke patient is shown in Fig. 3b, in which the difference in the vector density and arrangement as well as the distortion and time elongation of the force envelope are apparent. Thirty minutes after application of the anesthetic to the affected limb (Fig. 3c), the force diagrams assumed a more normal shape. The abnormal trial of force vectors that appeared at the end of the stance phase of the stroke patient was abolished after anesthesia.

Videotape assessment of the walking pattern provided qualitative evidence of a noticeable improvement in the heel-toe walking sequence and speed and smoothness of movement. Swing phases were less jerky during gait cycle, and weight acceptance was more controlled during the stance phase. Angular displacement at hip, knee, and ankle joints during ambulation showed a substantial increase in the range of movement after anesthesia. This finding confirmed our previous observation, which also showed the angular displacement to be increased significantly in amplitude either during performance of active movements or when the joint was moved passively $(11,12,14-16)$.
Videotape studies of the active movement patterns of the upper limb in simulated activities of daily living (ADL) showed an increase in the range of movement after anesthesia. Active shoulder abduction and flexion movements increased by as much as $65^{\circ}$ in some patients, and all patients showed some increase. Active movement became smoother, and patients were able to sustain muscle contraction for a longer time through the increased range of movement, indicating improvement in control of the paretic limb. The improved performance was more evident in the proximal rather than the distal limb joints.

This study documents the quantified results of patients who were able to complete the arduous 2month procedure. To date, however, we have subjectively observed short-duration improvements in limb mobility and/or diminution of muscle rigidity in numerous other spastic patients as a consequence of a single application of the topical anesthetic. These include 12 additional CVA patients, 5 Parkinson's disease patients, 8 cerebral palsy children (ranging in age from 10 months to 16 years), and 3 patients with spinal cord injuries.

\section{Long-Term Effects}

When the topical anesthetic was applied before every physical therapy session, it was easier for the 
TABLE 1. Immediate and long-term effects of topical anesthetic and placebo skin application on temporal components of gait in seven spastic patients

\begin{tabular}{|c|c|c|c|c|c|c|}
\hline \multirow[b]{2}{*}{ Patient } & \multicolumn{6}{|c|}{ Temporal components of gait } \\
\hline & $\mathrm{SF}$ & TAI & SLS & DSA & DSN & CAD \\
\hline \multicolumn{7}{|c|}{ Immediate effect of topical anesthesia } \\
\hline A.R. & - & - & $+{ }^{a}$ & & + & \\
\hline S.C. & + & & & + & & + \\
\hline B.F. & $t^{a}$ & + & $+^{a}$ & $t^{*}$ & $t^{*}$ & $++^{a}$ \\
\hline J.S. & $t^{a}$ & + & & $t^{*}$ & & $+^{a}$ \\
\hline D.G. & + & & & & & $+{ }^{a}$ \\
\hline M.B. & $t^{a}$ & + & & + & & \\
\hline E.H. & + & + & $+^{a}$ & + & $t^{a}$ & \\
\hline \multicolumn{7}{|c|}{ Long-term effect of topical anesthesia } \\
\hline A.R. & + & - & + & $t^{a}$ & $+^{a}$ & $t^{a}$ \\
\hline S.C. & + & $+^{a}$ & $+{ }^{a}$ & $+^{a}$ & $+^{a}$ & $+{ }^{a}$ \\
\hline B.F. & $+{ }^{a}$ & $+a$ & $+^{a}$ & $+{ }^{a}$ & $+{ }^{a}$ & $+a$ \\
\hline J.S. & + & + & & $+a$ & & \\
\hline D.G. & $+t^{a}$ & $+{ }^{a}$ & $+^{a}$ & & & $+^{a}$ \\
\hline M.B. & + & $+^{a}$ & $t^{a}$ & $+^{a}$ & + & + \\
\hline E.H. & + & & & $+^{a}$ & + & + \\
\hline \multicolumn{7}{|c|}{ Immediate effect of placebo } \\
\hline A.R. & & - & & & & \\
\hline S.C. & & & & - & & \\
\hline B.F. & & - & & - & $-a$ & \\
\hline J.S. & & & & - & & + \\
\hline D.G. & + & $+^{a}$ & & & & $+a$ \\
\hline M.B. & - & + & & $-{ }^{a}$ & & \\
\hline E.H. & - & - & & - & $t^{a}$ & + \\
\hline \multicolumn{7}{|c|}{ Long-term effect of placebo } \\
\hline $\begin{array}{l}\text { A.R. } \\
\text { S.C. }\end{array}$ & + & - & $t^{a}$ & $+^{a}$ & $t^{a}$ & + \\
\hline B.F. & $-a$ & & & $-{ }^{a}$ & + & \\
\hline J.S. & & & & $+^{a}$ & & $t^{a}$ \\
\hline D.G. & & $-{ }^{a}$ & $-a$ & & & \\
\hline M.B. & $-{ }^{a}$ & $-{ }^{a}$ & & & $t^{a}$ & \\
\hline E.H. & + & $-{ }^{a}$ & & & & \\
\hline
\end{tabular}

Modification in the parameter was toward normal standards $(+)$, a shift away from normal $(-)$, and no measurable change ( ).

${ }^{a}$ Modification was statistically significant $(\mathrm{p}<0.05)$.

SF, symmetry factor; TAI, temporal symmetry index; SLS, single limb support time; DSA, double support time on affected and (DSN) nonaffected limb; CAD, cadence.

patient and therapist to conduct the exercise program, apparently owing to the decreased muscular rigidity. Therapists reported a decrease in the stiffness of the affected muscles during passive stretch with a decreased abnomal active-movement synergy in most patients. The improved performance of active movement with reduction of pathologic synergy augmented the functional capability of the patients. The compound effect of reduced muscular rigidity and increased motor output is supported by our studies on the $\mathrm{H}$ and ATRs in the same patients $(15,16)$ and by our previous studies of normal subjects $(10,11)$.

At the end of the 1-month period of the topical
TABLE 2. Immediate and long-term effects of topical anesthetic and placebo skin application on time required to produce 10 RRM of knee and elbow joints in nine spastic patients

\begin{tabular}{|c|c|c|c|c|}
\hline \multirow[b]{2}{*}{ Patient } & \multicolumn{2}{|c|}{ Knee joint } & \multicolumn{2}{|c|}{ Elbow joint } \\
\hline & $45 \mathrm{~min}$ & Long-term & $45 \mathrm{~min}$ & Long-term \\
\hline \multicolumn{5}{|c|}{$\begin{array}{l}\text { Effect of topical anesthesia on knee } \\
\text { and elbow joint active excursion }\end{array}$} \\
\hline A.R. & $t^{a}$ & $+^{a}$ & + & + \\
\hline S.C. & $+^{a}$ & & + & - \\
\hline B.F. & $+^{a}$ & + & & \\
\hline $\begin{array}{l}\text { J.S. } \\
\text { D.G. }\end{array}$ & $+^{a}$ & $+{ }^{a}$ & $+{ }^{a}$ & $+{ }^{a}$ \\
\hline M.B. & $+^{a}$ & $+^{a}$ & $t^{*}$ & $t^{a}$ \\
\hline E.H. & $t^{a}$ & $+^{a}$ & $+^{*}$ & $t^{a}$ \\
\hline G.B. & + & $t^{a}$ & + & $t^{a}$ \\
\hline W.F. & $++^{a}$ & $t^{a}$ & + & \\
\hline \multicolumn{5}{|c|}{$\begin{array}{l}\text { Effect on placebo on knee and elbow joint } \\
\text { active excursion movement }\end{array}$} \\
\hline $\begin{array}{l}\text { A.R. } \\
\text { S.C. }\end{array}$ & & + & & \\
\hline B.F. & & $t^{a}$ & & \\
\hline $\begin{array}{l}\text { J.S. } \\
\text { D.G. }\end{array}$ & & $-{ }^{a}$ & & + \\
\hline M.B. & & & + & \\
\hline E.H. & & - & & + \\
\hline $\begin{array}{l}\text { G.B. } \\
\text { W.F. }\end{array}$ & & & + & \\
\hline
\end{tabular}

Modification in the required time was toward normal values $(+)$, a shift away from normal $(-)$, and no measurable change ( ).

${ }^{a}$ Modification was statistically significant $(\mathrm{p}<0.05$ ).

anesthetic program, improvement in movement parameters was noted in all patients, as shown in Fig. 1. Time measurements of the 10 RRM demonstrated a progressive reduction in performance time. This patient completed $10 \mathrm{RRM}$ at the elbow joint in $20 \mathrm{~s}$ at the beginning of the study, whereas at the end of 1 month of anesthetic treatment he required only 13 $\mathrm{s}$, a $35 \%$ increase in movement velocity. When we performed a $t$ test between days 1 and 30 , we noted a statistically significant $(\mathrm{p}<0.05)$ reduction in movement time. Figure 2 shows similar results in another patient tested for the time performance of 10 RRM at the knee joint. The patient completed the $10 \mathrm{RRM}$ at the knee joint in $17 \mathrm{~s}$ at the beginning of the study, whereas at the end of 1 month of anesthetic treatment he required only $10 \mathrm{~s}$ to complete the task. This shows a statistically significant ( $\mathrm{p}<$ 0.001 ) long-term reduction in the time required for completion of 10 RRM. A $t$ test performed between the beginning and end of the month of the placebo showed no statistically significant difference in the measured parameter. 
FORCE VECTOR DIAGRAMS

a) $\frac{x}{\alpha}$

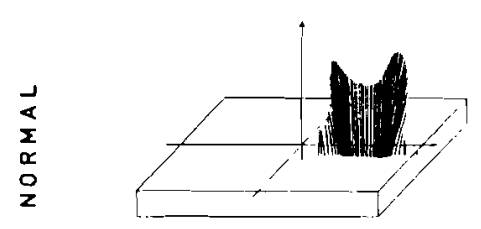

b)

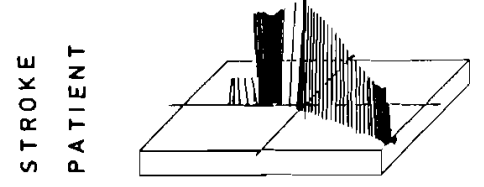

c
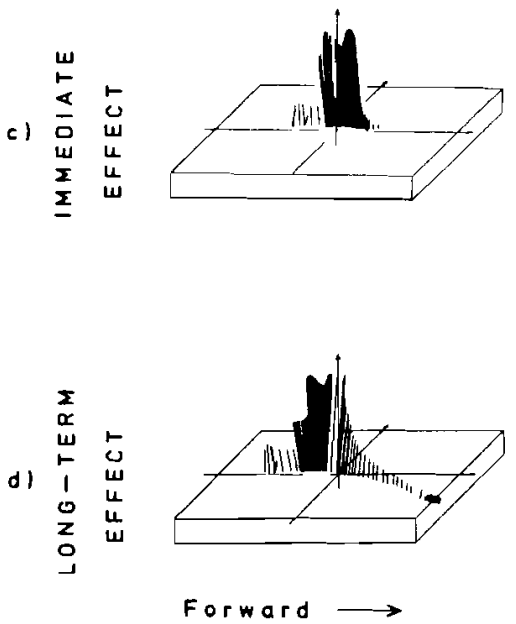

FORCE ENVELOPE
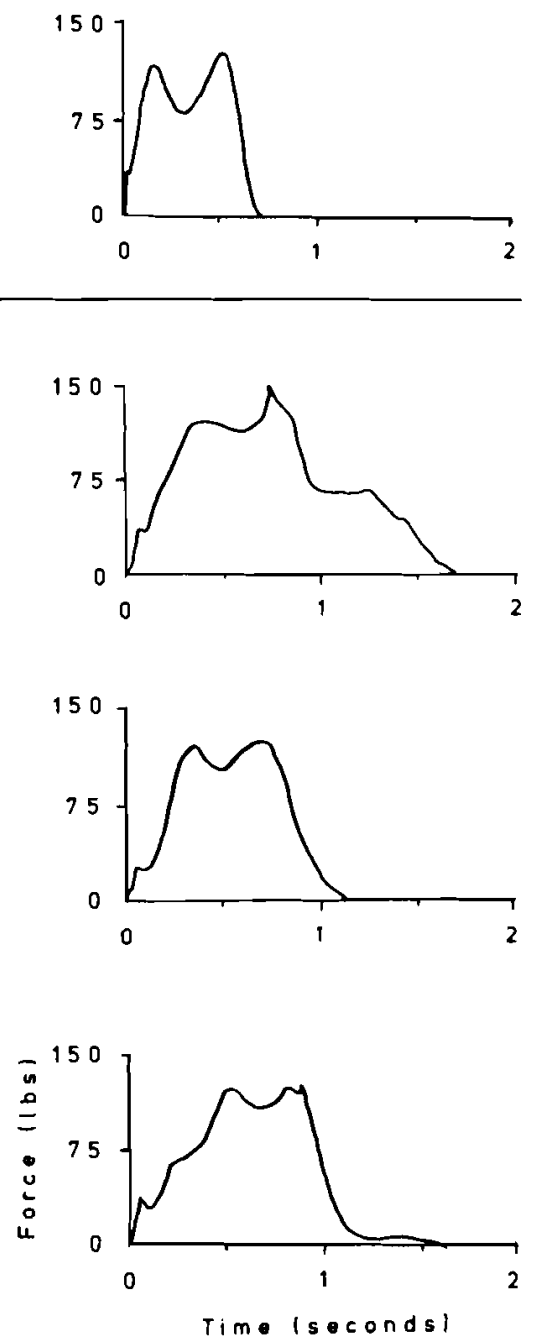

FIG. 3. Vector diagrams (left) and magnitude envelope (rlght) of vertical force measured by the force plate as a subject (63-year-old hemiplegic patient) moved forward over the force plate from the time of heel strike to toe-off. a: Typical curves for a normal subject. b: Curves for a stroke patient. The dissimilarity is apparent. c: Curves for the patient in b $45 \mathrm{~min}$ after first application of topical anesthetic (improvement in comparison with the normal subject is apparent). d: Curves for the patient in $b$ and $c$ at the end of the 1-month topical anesthesia and physical therapy program. Better foot clearance at the end of the stance phase is evident. This residual modification is indicative of long-lasting improvement.
One might argue that the changes in movemment performance shown in Figs. 1 and 2 resulted from waxing and waning of the spasticity. Although this is an inherent problem in patients affected with spasticity, it did not affect our results dramatically for two reasons: First, each patient was tested for the immediate (short-term) effect during the same recording session when no extraneous factors influenced the patient's muscle tone. Second, the longterm effect was indicated by the trend in movement performance during the month of anesthesia or placebo treatments. Figures 1 and 2 show that such possible waxing and waning of spasticity was not apparent during the month of placebo application. On the contrary, variability in movement performance appeared to be relatively smaller during the month of placebo application as compared with the month of anesthetic application. Therefore, the cumulative long-term reduction of movement performance time during the first month appears to be due to application of the topical anesthetic and not to other extraneous factors.

The gait parameters for all patients (Table 1) indicate that almost every parameter of the temporal components of the gait cycle showed a statistically significant $(\mathrm{p}<0.05)$ shift toward normal standards. In the sample subject (Fig. 1), single limb support time and cadence improved by $35 \%$. The vertical ground reaction force assumed a more normal envelope. The abnormal trial of force vectors which appeared at the end of the stance phase of the stroke patient diminished considerably, indicating a 
FIG. 4. Comparison of the modifications of the gait (movement) parameters induced by application of topical anesthetic spray or a placebo spray on the skin surface in one representative patient. Measurements were taken 45 min after first application of the spray and at the end of a 1 -month combined topical-spray physical therapy program. In the latter case, the measurements were made without application of the spray. The temporal sequence of gait cycles was measured with the following variables: temporal asymmetry index (TAl), symmetry factor (SF), left double support (LDS), left single limb support (LSLS), and cadence (CAD). These data show a comparison between the short-term or immediate (hatched) and long-term (solid) effects of topical anesthetic (top) or placebo (bottom) on temporal gait parameters in a left hemiplegic patient. Percentage of improvement was calculated as the percentage of the shift in the value of the parameters toward normal standards. These data from a single subject (35-year-old head injury patient) represent the changes recorded in most of our patients. Degree of improvement varied among subjects. Circled L, hemiplegic.

decreased shuffling of the foot during ambulation. These results were also noted in the force-time curve.

Table 2 shows that this outcome was typical for eight of nine patients. A videotape study of upper and lower limb movement at the end of the treatment program demonstrated improvement in active ROM, movement speed and synergy, and heel-toe walking pattern during ambulation, and a near absence of foot shuffling during swing phase. These improvements in movement performance were reflected in the functional ADL as reported by the patients.

\section{DISCUSSION}

The modification of the movement parameters noted within $30-45 \mathrm{~min}$ of application of the topical anesthetic suggests that excitation and/or inhibition of the $\alpha$ and $\gamma$ systems were altered. The $\alpha$-motoneurons increased in excitability (resulting in increased motor output) and simultaneously the $\gamma$-motoneurons decreased in excitability (resulting in decreased muscular rigidity). These findings are consistent with our previous work on the $\mathrm{H}$ and ATR in normal human subjects $(10,13)$ and with the work of other investigators in animals $(2,3,19,20)$. A similar explanation was offered to account for the reduction of physiologic action tremors in normal subjects and pathologic clonus in spinal cord injury patients after desensitization of the skin by topical anesthesia (9). Other recent work of ours suggests

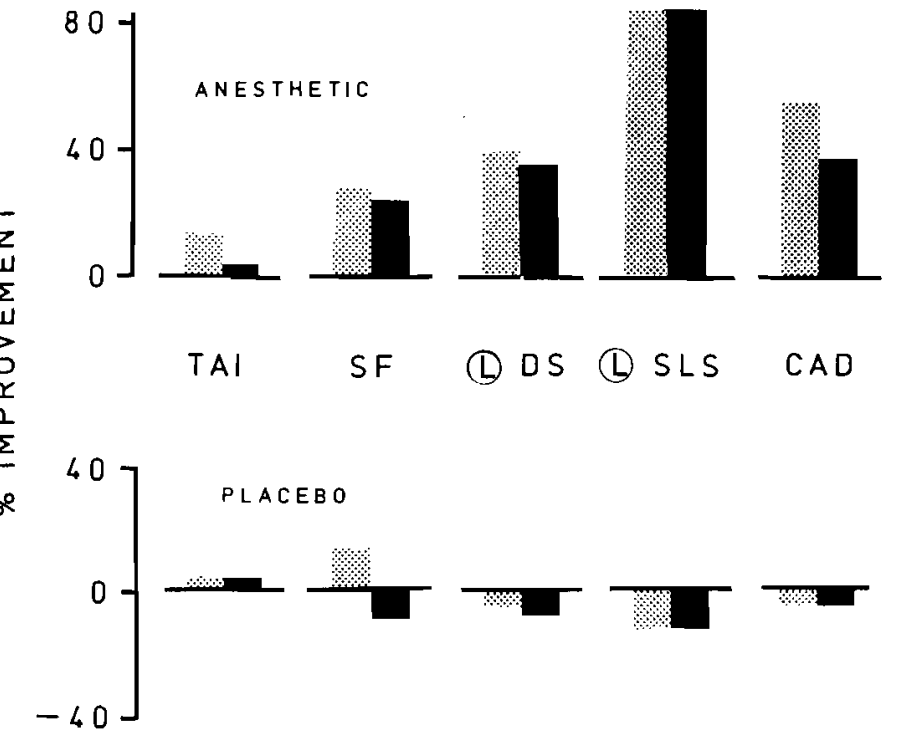

that removal of excitatory drive from the skin by application of topical anesthetic would favor increased inhibition in the spinal cord (8). This behavior could cause the previously suggested inhibition of $\alpha$-motoneurons (13) and tonic reflexes (17).

Because the improvements in the movement patterns and reduction in rigidity were not evident when the placebo spray was used, we inferred that the modifications in motor output are induced by the anesthetic agent. The effect may occur in at least two possible ways. First, it may be mediated by the direct effect on $\alpha$ - and $\gamma$-motoneurons of the anesthetic that is absorbed into the blood stream. Such a possibility is unlikely. In a companion study, we detected no traces of benzocaine in the systemic circulation after extensive application of topical anesthetic to the skin of the upper and lower limbs. Second, it may be mediated by the desensitizing effect on the cutaneous receptors. This latter explanation implies that the affected cutaneous receptors (either receptor adaptation or reduced activation) either generate spontaneous activity or are stimulated to discharge by the motion of the skin as the limb begins to move. Mechanoreceptors with group III or IV fibers have been shown to have both modalities of behavior $(4-6,18)$ and may be considered suitable candidates for either signaling or inducing the alterations in motor output, although other skin receptors may participate. Therefore, the reduced activity of the cutaneous receptors would induce a slow-acting accommodation in the peripheral motor system. 
Wolf and Mirkwitz reported no significant changes in the H-reflex or the ATR of stroke patients after benzocaine anesthetic. The difference in results in the study of Wolf and Mirkwitz (21) and our current studies may be due to different methodology as well as criteria applied to patient selection.

The long-term effect on the motor system observed in this study is a reflection of the potential overt plasticity of the CNS. The observed movement improvement is most likely due to the physical exercise that the patients were able to perform as a result of application of the topical anesthetic. The effects of the anesthetic lasted $\sim 3 \mathrm{~h}$ and peaked 30-45 min after application (10). When desensitization subsided, the movement pattern of the patients reverted to the preanesthetic state. Thus, application of the anesthetic was used to generate a time window during which patients were able to perform physical therapy exercise they could not otherwise perform. We believe that this augmented therapy induced the long-term effects. Although all the patients were chosen from a population that was no longer benefiting from its current physical therapy program, we were able to augment the limb movement range of all of them. In some patients, this augmented movement range gave them freedom to perform basic ADL such as eating, grooming, and hygiene maintenance on their own.

Acknowledgment: We thank our patients who endured an arduous routine and personal inconvenience in order to participate for the duration of the experiment. We thank Serge Roy and C. A. Van Volkinberg for expert assistance in the therapy program. This work was supported by Liberty Mutual Insurance Company.

\section{REFERENCES}

1. Dewar ME, Judge G: Temporal asymmetry as a gait indicator. Med Biol Eng Comput 18:689-693, 1980.

2. Ellaway PH, Murphy PR, Tripathi A: Group III muscle afferents which excite gamma motoneurons in the cat. In Proceedings of the Eleventh Annual Meeting of the Society for Neuroscience. Los Angeles: Society for Neuroscience, 7:188, 1981.

3. Golesz FA, Cheng-Tao X, Ruenzel PW, Henneman E:
Flexor reflex control of the external sphincter of the urethra in paraplegia. Science 216:1243-1245, 1982.

4. Johansson RS: Tactile sensibility in the human hand: receptive field characteristics of mechanoreceptive units in the glabrous skin area. J Physiol (London) 281:101-123, 1978.

5. Knibestol M, Vollbo AB: Single unit analysis of mechanoreceptor activity from the human glabrous skin. Acta Physiol Scand 80:178-195, 1970.

6. Knibestol M: Stimulus-response functions of slowly adapting mechano-receptors in the human glabrous skin area. $J$ Physiol (London) 254:63-80, 1975.

7. Knutsson E: An analysis of parkinsonian gait. Brain 95:475486, 1972.

8. Lou EC, Sabbahi MA: Topical anesthesia: effect of desensitization of the skin of the upper limb on tonic vibration reflex. Phys Ther 66:796, 1986.

9. Mills WJ, Pozos RS: A decrease in clonus amplitude by topical anesthesia. Electroencephalogr Clin Neurophysiol 61:509-518, 1985.

10. Sabbahi MA, De Luca CJ: Topical anesthesia: H-reflex recovery changes by desensitization of the skin. Electroencephalogr Clin Neurophysiol 52:328-335, 1981.

11. Sabbahi MA, De Luca CJ, Johnson LA: Topical anesthesia: a new method for improving motor control in spasticity. $J$ Am Phys Ther Assoc 61:735, 1981.

12. Sabbahi MA, De Luca CJ, Powers WR: Topical anesthesia: a possible treatment method for spasticity. Arch Phys Med Rehab 62:310-314, 1981.

13. Sabbahi MA, De Luca CJ: Topical anesthesia: modulation of the monosynaptic reflexes by desensitization of the skin. Electroencephalogr Clin Neurophysiol 54:677-688, 1982.

14. Sabbahi MA, De Luca CJ, Johnson LA: Topical anesthesia: A new treatment method for spasticity. Proceedings of the Ninth Congress of the World Confederation for Physical Therapy. Congress of the World Confederation for Physical Therapy, Stockholm, 1982, pp 601-604.

15. Sabbahi MA, Roy SH, De Luca CJ, Van Volkinberg LA: Topical anesthesia: changes in the control of movements in spastic patients. Proceedings of the 13th Annual Meeting of the Society for Neuroscience. Los Angeles: Society for Neuroscience, 1983; p 1037.

16. Sabbahi MA, Roy SH, De Luca CJ, Van Volkinberg LA: Active and passive range of joint movements in stroke patients after desensitization of the skin. Neurosci Abstr 10:905, 1984.

17. Sabbahi MA, Mason CR, Lou EC. Modulations of phasic and tonic reflexes with the skin. Neurosci Abstr 12:969, 1986.

18. Schaible MG, Schmidt RF: Activation of groups III and IV sensory units in medial articular nerve by local mechanical stimulation of knee joint. J Neurophysiol 49:35-44, 1983.

19. Schieppati AM, Crenna P: Natural cutaneous stimulation induces late and long-lasting facilitation of extensor motoneurons in the cat. Brain Res 293:259-267, 1984.

20. Seguin JJ, Cooke JD: The effects of cutaneous mechanoreceptor stimulation on the stretch reflex. Exp Brain Res 52:152-154, 1983.

21. Wolf SL, Mirkwitz JA: Topical anesthetics: effects on Achilles tendon and H-reflexes. II. Stroke patients. Arch Phys Med Rehabil 70:673-677, 1989. 